\title{
Pour une approche sémantique du lexique verbal en développement normal et pathologique : Entre linguistique et psychologie : une histoire de mots et/ou ? de catégorisation
}

\author{
Karine Duvignau, CLLE-ERSS UMR 5263, Université Toulouse 2 \& CNRS \\ duvignau@univ-tlse2.fr
}

\begin{abstract}
Lorsque placé à un niveau d'analyse sémantique, on examine les principales données en matière d'acquisition du lexique, il ressort que, depuis le développement lexical précoce chez l'enfant normal (acquisition précoce) jusqu'à la personne âgée atteinte de la Démence de Type d'Alzheimer (désacquisition) les travaux, tant en linguistique qu'en psychologie, portent massivement sur le lexique nominal au détriment de l'étude du verbe. En effet, même si on assiste depuis quelques années à un accroissement des approches lexico-sémantiques qui focalisent leur attention sur le verbe, il n'en reste pas moins que la littérature disponible pour un éventail large de la cognition humaine véhicule toujours l'hégémonie du nom. Nous illustrons cette situation en traversant différents champs de la linguistique et de la psycholinguistique via un phénomène crucial parce que charnière entre les mots et la catégorisation : le phénomène métaphore. Ce parcours consistera à interroger la nature/fonction d'énoncés d'allure métaphorique à pivot verbal :
\end{abstract}

- au cours du développement lexical précoce des verbes (jeunes enfants)

- chez des enfants atteints de Troubles Spécifiques du Langage

- lorsque l'organisation du lexique est relativement stable (adultes)

- en situation de déficit lexical (adultes locuteurs en Français Langue Etrangère)

- en situation de « dégradation » lexico-sémantique (Alzheimer)

- en situation de problème d'accès lexical (aphasique)

- lorsque le lexique est hors norme du fait de son extrême précision (syndrome d'Asperger)

- chez des autistes de haut niveau

Nous serons amenées à articuler métaphore et catégorisation et à retenir non pas le seul argument du choix lexical pour déterminer l'existence de métaphore mais des phénomènes linguistiques tels que le rejet ou la modalisation comme marqueurs de la condition de la métaphore à savoir : une (dé)catégorisation des mots en catégories distinctes. Ce trajet s'ouvrira sur des pistes de recherche qui traversent la linguistique et la psychologie : interroger le rapport entre flexibilité sémantique du système linguistique et la flexibilité cognitive des sujets et discuter de la pertinence, dans le domaine du verbe, du recours à la notion d'erreur actuellement de mise dans un grand nombre d'approches linguistiques psycholinguistiques ou cliniques. 